\title{
Single-Source Three-Disjoint Path Covers in Cubes of Connected Graphs
}

\author{
Jung-Heum Park ${ }^{\mathrm{a}}$, Insung $\operatorname{Ihm}^{\mathrm{b}, *}$ \\ ${ }^{a}$ School of Computer Science and Information Engineering \\ The Catholic University of Korea, Bucheon, Korea \\ ${ }^{b}$ Department of Computer Science and Engineering \\ Sogang University, Seoul, Korea
}

\begin{abstract}
A $k$-disjoint path cover ( $k$-DPC for short) of a graph is a set of $k$ internally vertex-disjoint paths from given sources to sinks that collectively cover every vertex in the graph. In this paper, we establish a necessary and sufficient condition for the cube of a connected graph to have a 3-DPC joining a single source to three sinks. We also show that the cube of a connected graph always has a 3-DPC joining arbitrary two vertices.
\end{abstract}

Keywords: Disjoint path cover, hamiltonicity, graph embedding, cube of a graph, combinatorial problems.

\section{Introduction}

Given an undirected graph $G$, a path cover of $G$ is a set of paths that collectively cover all vertices of $G$. It is called a disjoint path cover (DPC for short) if every vertex of $G$, possibly except terminal vertices, belongs to exactly one path. Given two disjoint terminal vertex sets $S=\{s\}$ and $T=\left\{t_{1}, t_{2}, \ldots, t_{k}\right\}$, a one-to-many $k$-disjoint path cover is a disjoint path cover made of $k$ paths, each joining a pair of source $s$ and sink $t_{i}, i=1, \ldots, k$. A graph $G$ is called one-to-many $k$-coverable if $|V(G)| \geq k+1$ and there exists a one-to-many $k$-DPC for any $S$ and $T$. When $S=\{s\}$ and $T=\{t\}$, a disjoint path cover made of $k$ paths, each joining $s$ and $t$ is named a one-toone $k$-disjoint path cover. A graph $G$ is said to be one-to-one $k$-coverable if

\footnotetext{
*Corresponding author

Email addresses: j.h.park@catholic.ac.kr (Jung-Heum Park), ihm@sogang.ac.kr (Insung Ihm)
} 
$|V(G)| \geq 2$ and there exists a one-to-one $k$-DPC for any $s$ and $t$. The other possible type of the disjoint path cover is a many-to-many $k$-disjoint path cover, whose $k$ disjoint paths collectively join two disjoint terminal vertex sets of cardinality $k$. Since this article deals only with the first two kinds of $k$-DPCs, refer to, for example, $[22,23]$ for details of the many-to-many $k$-DPC.

The disjoint path cover naturally arises in various applications such as software testing, database design, and code optimization [2, 19]. It is also deeply related to the full utilization of nodes in interconnection networks [22]. Several special classes of graphs have been studied in terms of the existence of desired disjoint path covers: hypercubes $[6,8,13]$, recursive circulants $[16,22,23]$, and hypercube-like graphs [22, 23]. The many-tomany 2-disjoint path cover problem with respect to the cubes of connected graphs was also studied recently [21].

Notice that the existence of a disjoint path cover for a given graph is greatly affected by its vertex connectivity. For instance, a graph is $k$ connected if and only if it has $k$ disjoint paths of many-to-many type, respectively connecting arbitrary $k$ distinct sources and arbitrary $k$ distinct sinks, where, if a source coincides with a sink, then such source itself is regarded as a path. Furthermore, Menger's theorem and the Fan Lemma indicate such strong relationship [3]. When the vertex connectivity is not high enough, one way of enhancing the probability of the existence of a disjoint path cover of a graph is to increase the connectivity by adding more edges: given a graph $G$ and a positive integer $d$, the $d$ th power $G^{d}$ of $G$ is the graph made of the vertex set $V(G)$ and the edge set $E\left(G^{d}\right)$, augmented in such a way that $(u, v) \in E\left(G^{d}\right)$ if and only if there is a path of length at most $d$ in $G$ joining $u$ and $v$. Particularly, $G^{2}$ are $G^{3}$ are called the square and the cube of $G$, respectively.

This paper aims to investigate the structure of the cubes of connected graphs with respect to one-to-many and one-to-one 3-disjoint path covers which we will collectively call as single-source 3-disjoint path covers. In particular, we establish a necessary and sufficient condition for the cube of a connected graph to have a 3 -DPC joining a single source to three sinks. Based on this result, we also show that the cube of every connected graph of order at least four is one-to-one 3-coverable.

\section{Preliminaries}

In addition to the vertex connectivity, the disjoint path cover is easily associated with the concept of hamiltonicity. A hamiltonian path between two 
vertices in a graph is actually a 1-DPC regardless of its type. By definition, a graph of order at least three is one-to-many 2-coverable if and only if it is hamiltonian-connected, where a graph is said to be hamiltonian-connected if every pair of vertices are joined by a hamiltonian path. Furthermore, a graph is one-to-one 2-coverable if and only if it is hamiltonian. A hamiltonian path/cycle passing through prescribed edges may also be constructed by finding a disjoint path cover as suggested in [21, 22, 23].

There have been extensive works on the hamiltonicity of graphs. Here, we briefly review those that are concerned with the squares and the cubes of graphs. It was shown that the square of every 2-connected graph is hamiltonian $[10,12]$. In fact, it is hamiltonian-connected and is 1-hamiltonian provided its order is at least four [4]. Several aspects of the hamiltonicity of the square of a connected graph were discussed in $[1,7,9]$. Also, some interesting properties on the pancyclicity and panconnectedness of the square of a connected graph were studied in [11]. Given a tree, its square was shown to be hamiltonian if and only if the tree is a caterpillar [14]. The square of a tree also has a hamiltonian path if and only if the tree is a horsetail as revealed in [24]. The fact that the cube of every connected graph is hamiltonianconnected was proved independently in $[26,15]$. It was also shown that the cube of a connected graph having order at least four is 1-hamiltonian [5]. Trees and connected graphs whose cubes are 1-hamiltonian-connected were characterized respectively in $[18,25]$, while connected graphs whose cubes are $p$-hamiltonian for $p \leq 3$ were classified in $[17,25]$. In [20], strong hamiltonian properties of the cube of a 2-edge-connected graph were also studied.

Before proceeding to our main results, we shall first introduce some basic terminologies and two fundamental properties of the cube of a connected graph. An edge of a graph $G$ is called a bridge if its removal increases the number of connected components (refer to Figure 1 for a pictorial illustration). A bridge is said to be nontrivial if neither of its two end vertices is of degree one. A vertex of $\mathrm{G}$ is called a pure bridge vertex if each of its incident edges is a nontrivial bridge. Furthermore, a set of three mutually adjacent vertices, each having a degree of at least three, is called a pure bridge triangle if every edge that is incident with exactly one of the triangular vertices is a nontrivial bridge.

Among several hamiltonian properties of the cubes of connected graphs, the following two play key roles in developing our results.

Lemma 1 (Sekanina [26] and Karaganis [15]). The cube of every connected graph is hamiltonian-connected. 


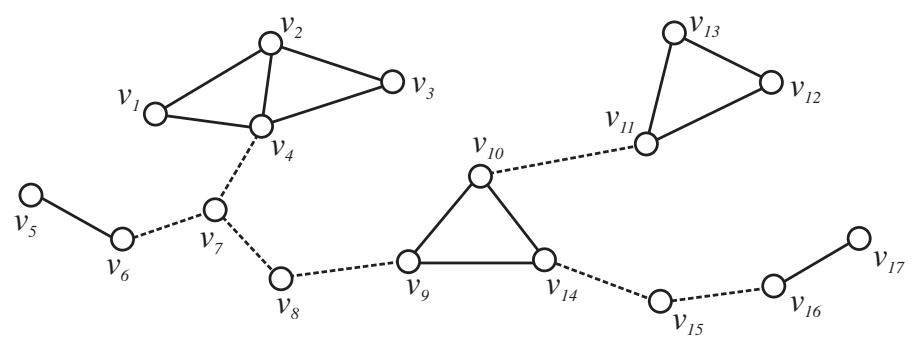

Figure 1: In this connected graph, the seven nontrivial bridges are represented by dotted lines. Also, there are three pure bridge vertices $v_{7}, v_{8}$, and $v_{15}$, and one pure bridge triangle $\left\{v_{9}, v_{10}, v_{14}\right\}$.

Lemma 2 (Schaar [25]). Given three vertices $s, t$, and $v_{f}$ of a connected graph $G$, there exists an $s$-t hamiltonian path in $G^{3} \backslash v_{f}$ if and only if (a) $\left\{s, t, v_{f}\right\} \nsubseteq N[v]$ for any pure bridge vertex $v$ of $G$ and $(b)\left\{s, t, v_{f}\right\}$ does not form a pure bridge triangle of $G$.

Here, $N_{G}(v)$, or $N(v)$ if the graph $G$ is clear in the context, denotes the open neighbor of vertex $v \in V(G)$, i.e. $N_{G}(v)=\{u \in V(G):(u, v) \in$ $E(G)\}$, while $N_{G}[v]$, or $N[v]$, represents the closed neighbor, i.e. $N_{G}[v]=$ $N_{G}(v) \cup\{v\}$. Then, we naturally define $N_{G}(X)=\bigcup_{v \in X} N_{G}(v) \backslash X$ and $N_{G}[X]=N_{G}(X) \cup X$ for a given vertex set $X \subseteq V(G)$. Also, the usual notations, $\delta_{G}(v)$ and $d_{G}(u, v)$, are used to denote the degree of vertex $v$ in $G$ and the distance between vertices $u$ and $v$ in $G$, respectively. Finally, we will call a vertex a nonterminal vertex if it does not belong to any terminal vertex set.

\section{Single-Source Three-Disjoint Path Covers}

From the fact that the cube of a connected graph is hamiltonianconnected, we easily see that it always has a one-to-many 2-DPC for any selection of source and sinks, meaning that it is one-to-many 2-coverable, and thus is one-to-one 2-coverable. Unlike the 2-DPC case, the cube of a connected graph contains a one-to-many 3-DPC only when certain conditions are met between terminal vertices. In this section, we make efforts to find the exact conditions that guarantee the existence of one-to-many 3 -DPC in the cube of a connected graph.

Lemma 3 (Necessity for one-to-many 3-DPC). Given a connected graph $G$, consider arbitrary terminal sets $S=\{s\}$ and $T=\left\{t_{1}, t_{2}, t_{3}\right\}$. 
If $G^{3}$ has a one-to-many 3-DPC joining $S$ and $T$, then $C 1$ : there exists no pure bridge vertex $v$ in $G$ such that $T \subseteq N_{G}[v]$ and $s \notin N_{G}[v]$, and C2: $T$ does not form a pure bridge triangle in $G$ such that $s \notin N_{G}[T]$.

Proof. Suppose that there exists a one-to-many 3-DPC in $G^{3}$ even though $T \subseteq N_{G}[v]$ and $s \notin N_{G}[v]$ for some pure bridge vertex $v$ of $G$ with $d \equiv$ $\delta_{G}(v) \geq 2$. Since $v$ is a pure bridge vertex, $G \backslash v$ has $d$ connected components, in which $s$ belongs to a component containing a neighbor of $v$, named $v_{1}$. Furthermore, for the other $d-1$ connected components, there is at least one nonterminal vertex per component that is not in $N_{G}(v) \backslash v_{1}$. Then, the three paths in the one-to-many 3-DPC originating from $s$ should collectively have at least $d-1$ edges in $G^{3}$, each ending up in such a vertex because each of the $d-1$ connected components must be visited. However, these edges can only start from vertices in the nonterminal set $N_{G}[v] \backslash T$ and its cardinality is $d-2$, we are led to a contradiction.

Suppose now that there exists a one-to-many 3-DPC in $G^{3}$ even though $T$ forms a pure bridge triangle in $G$ such that $s \notin N_{G}[T]$. Consider the graph $G \backslash\left\{\left(t_{1}, t_{2}\right),\left(t_{2}, t_{3}\right),\left(t_{3}, t_{1}\right)\right\}$, where we name the three connected components $G_{i}$ so as to $t_{i} \in V\left(G_{i}\right), i=1,2,3$, and assume w.l.o.g. that $s$ belongs to $G_{1}$. We first claim that no single path in the one-to-many 3-DPC passes through all the nonterminal vertices of $G_{2}$ and $G_{3}$. To prove it, assume, for a contradiction, that a path in the 3 -DPC does. Then, the path can be represented as $\left(x_{1}=s, x_{2}, \cdots, x_{l}=v_{i}, x_{l+1}=w, \cdots, x_{n}=t_{j}\right)$ for some sink $t_{j}$, where $w$ is the first nonterminal vertex in the path that is in $H$, the subgraph of $G$ induced by $V\left(G_{2}\right) \cup V\left(G_{3}\right)$, thus implying $v_{i} \in N_{G_{1}}\left(t_{1}\right)$ and $w \in N_{G_{2}}\left(t_{2}\right) \cup N_{G_{3}}\left(t_{3}\right)$. Now, since each of $t_{2}$ and $t_{3}$ is a pure bridge vertex in $H$, by Lemma 2 , there is neither a $w$ - $t_{2}$ hamiltonian path in $H^{3} \backslash t_{3}$ nor a $w$ - $t_{3}$ hamiltonian path in $H^{3} \backslash t_{2}$. Furthermore, for $H^{\prime}$, the subgraph of $G$ induced by $V(H) \cup\left\{t_{1}\right\}$, there does not exist a $w$ - $t_{1}$ hamiltonian path in $H^{\prime 3} \backslash\left\{t_{2}, t_{3}\right\}$ because, otherwise, both the two just mentioned hamiltonian paths would exist. This means that the tail of the path from $w,\left(w, x_{l+2}, \cdots, x_{n-1}, t_{j}\right)$, must contain at least one path segment, all of whose vertices are in $V\left(G_{1}\right) \backslash t_{1}$. Since removing such a path segment from the tail still forms a legitimate path in $G^{3}$, we can build an $s$ - $t_{j}$ path, by repeatedly deleting such a path segment, that, once arriving at $w$, traverses all and only the nonterminal vertices of $H$ before terminating at $t_{j}$. However, the existence of such a $w$ - $t_{j}$ tail leads to a contradiction to Lemma 2, proving our claim.

Now, assume that $G \backslash T$ has $d$ connected components, where $s$ belongs to a component containing a neighbor of $t_{1}$, named $v_{1}$. Similarly as before, for the remaining $d-1$ connected components, there exists at least one 


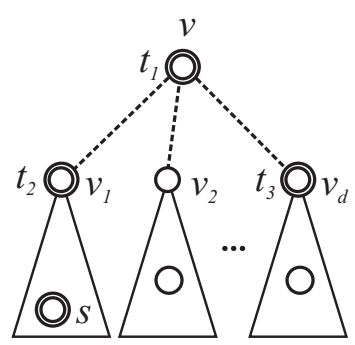

(a) Condition $\mathrm{C} 1$

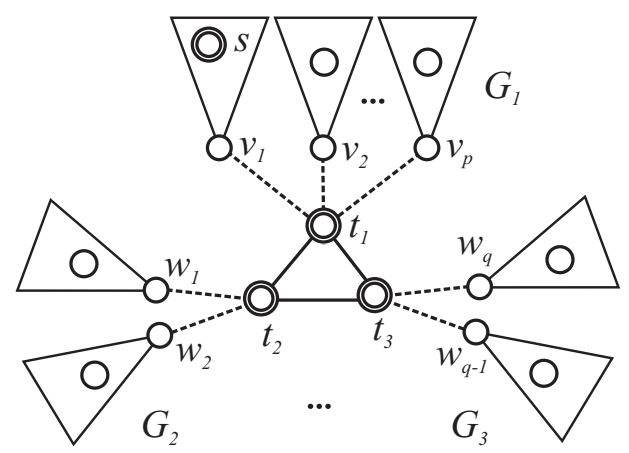

(b) Condition $\mathrm{C} 2(d=p+q)$

Figure 2: Illustration of the proof of Lemma 3.

nonterminal vertex per component that does not belong to $N_{G}(T) \backslash v_{1}$. Then, the three paths in the one-to-many 3-DPC should collectively have at least $d-1$ edges in $G^{3}$, each ending up in such a vertex because each of the $d-1$ connected components must be visited. Note that these edges can only start from vertices in the nonterminal set $N_{G}(T)$ whose cardinality is $d$. However, since at least two paths in the one-to-many 3-DPC must visit $H$, as implied by the claim made in the proof, at least two of the $d$ vertices must be consumed for connecting such paths, leaving at most $d-2$ free vertices in $N_{G}(T)$. Since we need at least $d-1$ free vertices, we have a contradiction. This completes the entire proof.

It is interesting to discover the fact that the two conditions, $\mathrm{C} 1$ and $\mathrm{C} 2$, in the above lemma also form the sufficient condition for the cube of a connected graph to have a one-to-many 3-DPC.

Theorem 1 (One-to-many 3-DPC). Given terminal sets $S=\{s\}$ and $T=\left\{t_{1}, t_{2}, t_{3}\right\}$ of a connected graph $G, G^{3}$ has a one-to-many 3-DPC joining $S$ and $T$ if and only if both the two conditions, C1 and C2, of Lemma 3 are satisfied.

Proof. The necessity part is due to Lemma 3. The sufficiency part proceeds by induction on the number of vertices of $G,|V(G)|$, assuming $|V(G)| \geq 5$ as the base case of $|V(G)|=4$ is trivial. The first step of the proof is to select a sink $t_{i}$ from $T$ such that all the other terminals in $\left\{s, t_{1}, t_{2}, t_{3}\right\} \backslash t_{i}$ exist in a same connected component of $G \backslash t_{i}$. Consider a 
spanning tree of $G$. Then, its smallest subtree containing all the four terminals have at least two leaves that are terminals. Since at least one of them should be a sink, we can always find a candidate for $t_{i}$. In case that there are more than one such sinks, $t_{i}$ is selected as follows: (i) choose the sink that has minimum number of incident edges that are not nontrivial bridges, and (ii) if there is still a tie between sinks, choose any of them that has maximum number of incident edges that are nontrivial bridges. Now, assume w.l.o.g. that $t_{1}$ has been selected. Then, let us denote by $H$ the connected component of $G \backslash t_{1}$ containing $s, t_{2}$, and $t_{3}$, and by $H^{\prime}$ the subgraph of $G$ induced by $V(G) \backslash V(H)$, where all the edges between the two disjoint subgraphs $H$ and $H^{\prime}$ are incident to $t_{1}$. In addition, let us define a vertex set $W$ as follows:

$$
W= \begin{cases}\left\{w \in V(H): d_{G}\left(t_{1}, w\right) \leq 2\right\} & \text { if }\left|V\left(H^{\prime}\right)\right| \geq 2, \\ \left\{w \in V(H): d_{G}\left(t_{1}, w\right) \leq 3\right\} & \text { if }\left|V\left(H^{\prime}\right)\right|=1, \text { i.e. } V\left(H^{\prime}\right)=\left\{t_{1}\right\} .\end{cases}
$$

The second step of the proof is to make a claim that, once proven, will immediately lead to a proof of the sufficiency part in a constructive way. The claim is that at least one of the following two statements is true: (i) $s \in W$, and (ii) there exists a nonterminal vertex $t_{1}^{\prime} \in W$ such that $S=\{s\}$ and the new sink set $\left\{t_{1}^{\prime}, t_{2}, t_{3}\right\}$ satisfy both the two conditions C1 and C2 with respect to $H$. Once this claim is proved, we can always construct a one-to-many 3-DPC of $G^{3}$ joining $S$ and $T$ as follows. First, consider a $v$ - $t_{1}$ hamiltonian path in $H^{\prime 3}$ for some neighbor $v$ of $t_{1}$ if $\left|V\left(H^{\prime}\right)\right| \geq 2$, which is guaranteed to exist by Lemma 1 , or a one-vertex path $t_{1}$ if $\left|V\left(H^{\prime}\right)=1\right|$. If $s \in W$, by the definition of the set $W$, we can build an $s-t_{1}$ path by connecting $s$ to the above path in $H^{\prime 3}$, forming a desired one-to-many 3DPC of $G^{3}$ together with a one-to-many 2-DPC of $H^{3}$ joining $\{s\}$ and $\left\{t_{2}, t_{3}\right\}$, which exists due to the fact that $H^{3}$ is hamiltonian-connected. If there is a nonterminal vertex $t_{1}^{\prime} \in W$ described in the statement (ii), by the induction hypothesis, there is a one-to-many 3-DPC of $H^{3}$, joining $\{s\}$ and $\left\{t_{1}^{\prime}, t_{2}, t_{3}\right\}$. Then, by replacing the $s$ - $t_{1}^{\prime}$ path with the $s$ - $t_{1}$ path, obtained by combining the $s-t_{1}^{\prime}$ path with the path covering $H^{\prime 3}$, we get a desired result.

The final step of the proof is to prove the claim. Suppose, for a contradiction, that (a) $s \notin W$, and (b) for every nonterminal vertex $w \in W$, if any, $\{s\}$ and $\left\{w, t_{2}, t_{3}\right\}$ break either of the two conditions with respect to $H$ (note that the two conditions cannot be violated simultaneously because no triple of vertices that form a pure bridge triangle can be contained in the closed neighbor of a pure bridge vertex). Then, there are three cases:

Case 1: For a nonterminal vertex $w \in W,\{s\}$ and $\left\{w, t_{2}, t_{3}\right\}$ violates $C 1$ with respect to $H$. In this case, $\left\{w, t_{2}, t_{3}\right\} \subseteq N_{H}[v]$ and $s \notin N_{H}[v]$ for 


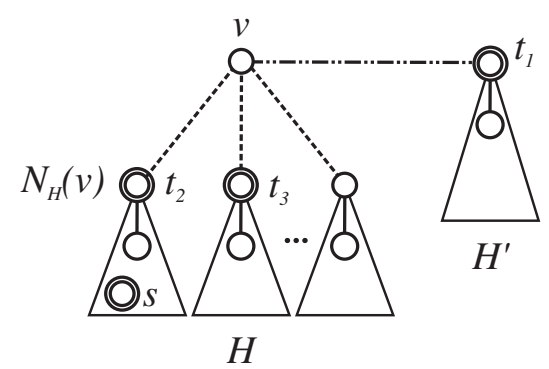

(a) $Z=\{v\}$

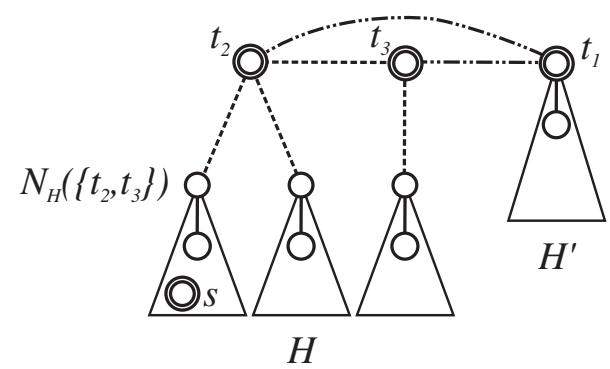

(b) $Z=\left\{t_{2}, t_{3}\right\}$

Figure 3: Illustration of Case 1 in the proof of Theorem 1.

some pure bridge vertex $v$ of $H$. Let $Z$ be the set of all pure bridge vertices $z$ such that $\left\{t_{2}, t_{3}\right\} \subseteq N_{H}[z]$. We can see that (i) if $v \notin\left\{t_{2}, t_{3}\right\}$, then $Z=\{v\}$, and (ii) if $v \in\left\{t_{2}, t_{3}\right\}$, either $Z=\{v\}$ or $Z=\left\{t_{2}, t_{3}\right\}$, depending on whether both $t_{2}$ and $t_{3}$ are pure bridge vertices or not. First, consider the case of $|Z|=1$, i.e. $Z=\{v\}$ whether $v \in\left\{t_{2}, t_{3}\right\}$ or not (Figure 3a). Every edge of $G$ from $t_{1}$ to $H$ may have the other end in either $V(H) \backslash N_{H}[v], N_{H}(v)$, or $\{v\}$. For an edge $\left(t_{1}, u\right) \in E(G)$ with $u \in V(H) \backslash N_{H}[v]$, which cannot be $s$ due to the hypothesis (a), $\{s\}$ and $\left\{u, t_{2}, t_{3}\right\}$ become to satisfy $\mathrm{C} 1$ and $\mathrm{C} 2$ for the nonterminal vertex $u \in W$, which contradicts the hypothesis (b). For an edge $\left(t_{1}, u\right) \in E(G)$ with $u \in N_{H}(v)$, since $v$ is a pure bridge vertex, there must be an edge $\left(u, u^{\prime}\right)$ such that $u^{\prime} \in V(H) \backslash N_{H}[v]$ and thus $u^{\prime}(\neq s) \in W$, which also leads to a contradiction to (b) with the sink set $\left\{u^{\prime}, t_{2}, t_{3}\right\}$. So, the only possibility left is that $\left(t_{1}, v\right) \in E(G)$ is the unique edge from $t_{1}$ to $H$, where $\left|V\left(H^{\prime}\right)\right| \geq 2$ because, otherwise, there would exist a nonterminal vertex $u^{\prime} \in V(H) \backslash N_{H}[v]$ with $u^{\prime}(\neq s) \in W$, which would cause the same contradiction. Hence, $\left(v, t_{1}\right)$ is a nontrivial bridge of $G$, implying that $v$ is in fact a pure bridge vertex of $G$ such that $T \subseteq N_{G}[v]$ and $s \notin N_{G}[v]$. This contradicts to the condition $\mathrm{C} 1$ of the theorem.

Second, consider the next case of $|Z|=2$, i.e. $Z=\left\{t_{2}, t_{3}\right\}$, where both $t_{2}$ and $t_{3}$ are pure bridge vertices (Figure $3 \mathrm{~b}$ ). Now, every edge of $G$ from $t_{1}$ to $H$ may have the other end in either $V(H) \backslash N_{H}\left[\left\{t_{2}, t_{3}\right\}\right], N_{H}\left(\left\{t_{2}, t_{3}\right\}\right)$, or $\left\{t_{2}, t_{3}\right\}$. For an edge $\left(t_{1}, u\right) \in E(G)$ for some $u$ in the first two vertex sets, the same reasoning as above leads us to a contradiction. So, the only possible edges from $t_{1}$ to $H$ are $\left(t_{1}, t_{2}\right)$ and $\left(t_{1}, t_{3}\right)$. Suppose that only one such edge $\left(t_{1}, t_{j}\right)$ exists for some $j=2,3$. Then, if $\left|V\left(H^{\prime}\right)\right|=1$, there always exists a vertex $u^{\prime} \in V(H) \backslash N_{H}\left[\left\{t_{2}, t_{3}\right\}\right]$ with $d_{G}\left(t_{1}, u^{\prime}\right)=3$ and thus $u^{\prime}(\neq s) \in W$, such that the sink set $\left\{u^{\prime}, t_{2}, t_{3}\right\}$ leads to a contradiction to (b) again. If 
$\left|V\left(H^{\prime}\right)\right| \geq 2, t_{j}$ is a pure bridge vertex of $G$ such that $T \subseteq N_{G}\left[t_{j}\right]$ and $s \notin N_{G}\left[t_{j}\right]$, contradicting the hypothesis of the theorem. Finally, suppose that both $\left(t_{1}, t_{2}\right)$ and $\left(t_{1}, t_{3}\right)$ exist. In this case, since $t_{1}, t_{2}$, and $t_{3}$ form a triangle in $G, t_{2}$ or $t_{3}$ also might have been chosen instead of $t_{1}$ in the first step of this proof. Since $t_{1}$ was chosen, $t_{1}$ should have exactly two incident edges that are not nontrivial bridges of $G$ and at least one incident edge that is a nontrivial bridge of $G$. This implies that $T$ forms a pure bridge triangle of $G$ and $s \notin N_{G}[T]$ due to (a), contradicting the condition C2 of the theorem.

Case 2: For a nonterminal vertex $w \in W,\{s\}$ and $\left\{w, t_{2}, t_{3}\right\}$ violates C2 with respect to $H$. In this case, $\left\{w, t_{2}, t_{3}\right\}$ forms a pure bridge triangle of $H$ such that $s \notin N_{H}\left[\left\{w, t_{2}, t_{3}\right\}\right]$. Clearly, it is impossible to have a pure bridge vertex $z$ of $H$ such that $\left\{t_{2}, t_{3}\right\} \subseteq N_{H}[z]$. Therefore, for any nonterminal vertex $w^{\prime} \in V(H),\{s\}$ and $\left\{w^{\prime}, t_{2}, t_{3}\right\}$ satisfy the condition C1. Furthermore, for a neighbor $u \in V(H)$ of $t_{1}$, define $u^{\prime}$ to be a neighbor of $u$ in $V(H) \backslash\left\{w, t_{2}, t_{3}\right\}$ if $u \in\left\{w, t_{2}, t_{3}\right\}$, or $u$ itself, otherwise. Then, since $u^{\prime}(\neq w)$ may not form a pure bridge triangle in $H$ with $t_{2}$ and $t_{3}$, for the vertex $u^{\prime}(\neq s) \in W,\{s\}$ and $\left\{u^{\prime}, t_{2}, t_{3}\right\}$ satisfy the condition C2 as well as C1, which contradicts (b).

Case 3: There exists no nonterminal vertex in $W$. The definition of $W$ entails that $|W| \geq 2$ if $\left|V\left(H^{\prime}\right)\right| \geq 2$, or $|W| \geq 3$, otherwise. Since $s \notin W$ due to (a), we have that $\left|V\left(H^{\prime}\right)\right| \geq 2$ and $W=\left\{t_{2}, t_{3}\right\}$. Furthermore, if $\left(t_{1}, t_{2}\right) \in$ $E(G)$, then $\delta_{G}\left(t_{2}\right)=2$ with $\left(t_{2}, t_{3}\right) \in E(G)$, and $\left(t_{1}, t_{3}\right) \notin E(G)$ because, otherwise, $W$ must contain at least one extra vertex with distance two from $t_{1}$. Similarly, if $\left(t_{1}, t_{3}\right) \in E(G)$, then $\delta_{G}\left(t_{3}\right)=2$ with $\left(t_{3}, t_{2}\right) \in E(G)$, and $\left(t_{1}, t_{2}\right) \notin E(G)$. In either case, there exists a pure bridge vertex $v$ of $G$ such that $T \subseteq N_{G}[v]$ and $s \notin N_{G}[v]$, which contradicts the condition C1 of the theorem. This completes the entire proof.

Corollary 1. For a connected graph $G$ with four or more vertices, $G^{3}$ is one-to-many 3-coverable if and only if there exists neither a pure bridge vertex nor a pure bridge triangle in $G$.

From Theorem 1, we can derive another interesting fact that the cube of a connected graph always has a one-to-one 3-DPC for any selection of source and sink.

Theorem 2 (One-to-one 3-DPC). For every connected graph $G$ with four or more vertices, $G^{3}$ is one-to-one 3 -coverable. 
Proof. Given a pair of source $s$ and sink $t$, define a vertex set $W=\{w \in$ $\left.V(G): 1 \leq d_{G}(t, w) \leq 3\right\}$, where it is obvious that $|W| \geq 3$. We claim that we can always find two nonterminal vertices $t_{2}$ and $t_{3}$ in $W$ such that $\{s\}$ and $\left\{t, t_{2}, t_{3}\right\}$ satisfy the two conditions in Theorem 1. If the claim is true, any one-to-many 3-DPC of $G^{3}$ joining these terminal sets may easily be transformed into a one-to-one 3 -DPC of $G^{3}$ by extending the $s$ - $t_{2}$ and $s$ - $t_{3}$ paths to $t$, proving the theorem. For a proof of the claim, we consider three classes of cases that embrace all cases. Firstly, if no edge incident to $t$ is a nontrivial bridge, it suffices to choose any two vertices in $W$ other than $s$ because $t$ must be incident to at least one nontrivial bridge to break any of the conditions of Theorem 1 . Secondly, if every edge incident to $t$ is a nontrivial bridge, $t$ has two neighbors, $u$ and $v$, which have respective neighbors $u^{\prime}$ and $v^{\prime}$ other than $t$. Then, by selecting $u$ and $v^{\prime}$ if $s \notin\left\{u, v^{\prime}\right\}$, or $u^{\prime}$ and $v$ otherwise, the two conditions in Theorem 1 can be satisfied. Finally, in the remaining class, there always exist two neighbors of $t, u$ and $v$, such that $(t, u)$ is a nontrivial bridge whereas $(t, v)$ is not a nontrivial bridge. If $s \notin\{u, v\}$, it is enough to choose $u$ and $v$. If $s \in\{u, v\}$, we consider a neighbor $u^{\prime}(\neq t)$ of $u$. If $u$ is not a pure bridge vertex, we choose the two vertices in $\left\{u, v, u^{\prime}\right\} \backslash s$. Otherwise, we select the two vertices in $\left\{u, v, u^{\prime \prime}\right\} \backslash s$ for some neighbor $u^{\prime \prime}(\neq u)$ of $u^{\prime}$. In any case, it is clear that the two conditions in Theorem 1 are satisfied.

\section{Concluding Remarks}

In this article, we have established a necessary and sufficient condition for the cube of a connected graph to have a one-to-many 3-DPC joining given terminal sets. We have also shown that the cube of every connected graph of order at least four is one-to-one 3 -coverable. The proofs are constructive, and hence may be used effectively to design a divide-and-conquer algorithm for finding a one-to-many 3-DPC, which can easily be modified to build a one-to-one 3-DPC through a simple reduction process. Another class of graphs that exposes an interesting connectivity structure is the square of a 2-connected graph. We conjecture that every such graph is one-to-many 3 -coverable, which remains to be proved in future work.

\section{Acknowledgement}

This research was supported by Basic Science Research Program through the National Research Foundation of Korea (NRF) funded by the Ministry of Education, Science and Technology (grant no. 2012R1A1A2005511 and 
2012R1A1A2008958). This work was partially supported by the Catholic University of Korea, Research Fund, 2012.

\section{References}

[1] M.E.K. Abderrezzak, E. Flandrin, and Z. Ryjáček, "Induced $S\left(K_{1,3}\right)$ and hamiltonian cycles in the square of a graph," Discrete Mathematics 207(1), pp. 263-269, 1999.

[2] K. Asdre and S.D. Nikolopoulos, "The 1-fixed-endpoint path cover problem is polynomial on interval graphs," Algorithmica 58(3), pp. 679-710, 2010.

[3] J.A. Bondy and U.S.R. Murty, Graph Theory, 2nd printing, Springer, 2008.

[4] G. Chartrand, A.M. Hobbs, H.A. Jung, S.F. Kapoor and C.St.J.A. Nash-Williams, "The square of a block is hamiltonian connected," Journal of Combinatorial Theory Ser. B 16, pp. 290-292, 1974.

[5] G. Chartrand and S.F. Kapoor, "The cube of every connected graph is 1-hamiltonian," J. Res. Nat. Bur. Standards 73B, pp. 47-48, 1969.

[6] X.-B. Chen, "Many-to-many disjoint paths in faulty hypercubes," Information Sciences 179(18), pp. 3110-3115, 2009.

[7] G.L. Chia, S.-H. Ong, and L.Y. Tan, "On graphs whose square have strong hamiltonian properties," Discrete Mathematics 309(13), pp. 4608-4613, July 2009.

[8] T. Dvořák and P. Gregor, "Partitions of faulty hypercubes into paths with prescribed endvertices," SIAM J. Discrete Mathematics 22(4), pp. 1448-1461, 2008.

[9] J. Ekstein "Hamiltonian cycles in the square of a graph," The Electronic Journal of Combinatorics 18(1), 2011.

[10] H. Fleischner, "The square of every two-connected graph is hamiltonian," Journal of Combinatorial Theory Ser. B 16, pp. 29-34, 1974.

[11] H. Fleischner, "In the square of graphs, hamiltonicity and pancyclicity, hamiltonian connectedness and panconnectedness are equivalent concepts," Monatshefte für Mathematik 82, pp. 125-149, 1976. 
[12] A. Georgakopoulos, "A short proof of Fleischner's theorem," Discrete Mathematics 309, pp. 6632-6634, 2009.

[13] P. Gregor and T. Dvořák, "Path partitions of hypercubes," Inform. Proc. Lett. 108(6), pp. 402-406, 2008.

[14] F. Harary and A. Schwenk, "Trees with hamiltonian square," Mathematika 18, pp. 138-140, 1971.

[15] J.J. Karaganis, "On the cube of a graph," Canad. Math. Bull. 11, pp. 295-296, 1968.

[16] S.-Y. Kim, J.-H. Lee, and J.-H. Park "Disjoint path covers in recursive circulants $G\left(2^{m}, 4\right)$ with faulty elements," Theoretical Computer Science, 412(35), pp. 4636-4649, 2011.

[17] K.M. Koh and K.L. Teo, "The 2-hamiltonian cubes of graphs," Journal of Graph Theory 13(6), pp. 737-747, 1989.

[18] L. Lesniak, "Graphs with 1-hamiltonian-connected cubes," Journal of Combinatorial Theory Ser. B 14(2), pp. 148-152, 1973.

[19] S.C. Ntafos and S.L. Hakimi, "On path cover problems in digraphs and applications to program testing," IEEE Trans. Software Eng. 5(5), pp. 520-529, 1979.

[20] M. Paoli, "Hamiltonian properties of the cube of a 2-edge connected graph," Journal of Graph Theory 12(1), pp. 85-94, 1988.

[21] J.-H. Park and I. Ihm, "Disjoint path covers in cubes of connected graphs," manuscript, 2012 (submitted for publication).

[22] J.-H. Park, H.-C. Kim, and H.-S. Lim, "Many-to-many disjoint path covers in hypercube-like interconnection networks with faulty elements," IEEE Trans. on Parallel and Distributed Systems 17(3), pp. 227-240, Mar. 2006.

[23] J.-H. Park, H.-C. Kim, and H.-S. Lim, "Many-to-many disjoint path covers in the presence of faulty elements," IEEE Trans. on Computers 58(4), pp. 528-540, Apr. 2009.

[24] J. Radoszewski and W. Rytter "Hamiltonian paths in the square of a tree," in Proc. Int'l Symp. Algorithms and Computation (ISAAC'11), pp. 90-99, 2011. 
[25] G. Schaar, "On 3-hamiltonian cubes of connected graphs," Colloquia Mathematica Societatis János Bolyai 18, pp. 959-971, 1978.

[26] M. Sekanina, "On an ordering of the set of vertices of a connected graph," Publ. Fac. Sci. Univ. Brno. 412, pp. 137-142, 1960. 